\title{
Final Report DE-FG07-97ER45671
}

July 2, 2004

(ID 59925) Modeling of Diffusion of Plutonium in Other Metals and of Gaseous Species in

$$
\text { Plutonium-Based Systems }
$$

Principal Investigator: Bernard R. Cooper

West Virginia University, Dept. of Physics, P.O. Box 6315, Morgantown, WV 26506-6315

304-293-3423, bcooper@wvu.edu

CoPrincipal Investigator: Gayanath W. Fernando

University of Connecticut, Physics Dept., Storrs, CT 06269

860-486-0442, fernando@cmtheory.phys.uconn.edu

Postdoctoral Research Associates: S. Beiden (year 1), A. Setty (subsequent period)

Graduate Student: E.H. Sevilla 


\title{
Final Report Special Considerations:
}

This report has been prepared on behalf of Professor Bernard R. Cooper by Department of Physics Chairman Professor Earl Scime in response to a request from the U.S. Department of Energy’s Procurement Services Division (Doreen Leonard, U.S. Department of Energy, Idaho Operations Office) for a final report. For some time now, Professor Cooper has suffered from a debilitating illness and is unable to complete the final report on this project at this time. Therefore, with his approval and with verbal approval from the U.S. Department of Energy, Professor Scime has prepared this report on behalf of Professor Cooper based on existing documentation concerning this report.

\author{
Earl Scime \\ Professor and Chair of Physics \\ West Virginia University \\ Morgantown, WV 26506
}

July 2, 2004 


\section{Research Objective:}

The problem being addressed is to establish standards for temperature conditions under which plutonium, uranium, or neptunium from nuclear wastes permeates steel, with which it is in contact, by diffusion processes. The primary focus is on plutonium because of the greater difficulties created by the peculiarities of face-centered-cubic-stabilized (delta) plutonium (the form used in the technology generating the waste).

Temperature is the key controllable diffusion process, i.e., temperature controls the rate of diffusion. The scientific goal of this project is to predict diffusion constants on an ab initio basis, i.e. diffusion distances in specified time at specified temperature for plutonium from plutonium-based waste materials into various steels or technologically-pertinent metallic alloys. This predictive ability will help to provide information relevant to setting temperature standards for maintaining structures, ducts, equipment, or waste-containing vessels until such time as decontamination and decommissioning and/or permanent storage can be carried out. In addition, this knowledge will aid in assessing the depth of penetration that must be dealt with in any surface treatment for decontamination.

The scientific steps of the methodology are (1) to recognize the stabilizing mechanism and the electronic structure pertinent to that stabilization for face-centered-cubic (fcc) deltastabilized plutonium, (2) to extract the information needed to perform dynamic simulations from ab initio electronic structure calculations, (3) to perform and report the dynamic simulations predicting the diffusion behavior.

\section{Research Progress and Implications:}

This final report summarizes the work completed on this 4-year project. We have 
completed step (1) recognizing the stabilizing mechanism and the electronic structure pertinent to that stabilization for face-centered-cubic (fcc) delta-stabilized plutonium; and the substantial computations and modeling for (2) and (3) are in progress and will be completed in a following study. The related experimental work is underway at Los Alamos National Laboratory, and the linkage been our work and that at LANL has been established in detail. In this report we summarize our successful identification of the electronic structure of $\delta$ plutonium and the validation in comparison with photoemission experiments carried out at Los Alamos.

The crystallographic behavior of plutonium has long been viewed as one of the most dramatic in the periodic table, going through a series of steplike expansions in atomic volume from the $\alpha$ monoclinic to the $\beta$ bodycentered monoclinic at 395K to the distorted diamond cubic $\gamma$ at $479 \mathrm{~K}$ to the cubic closepacked (fcc) $\delta$ at 592K, giving an overall expansion of atomic volume of about $27 \%$ between the $\alpha$ and $\delta$ structures. This behavior is widely viewed as a particularly striking example of the loss of $5 f$ bonding, i.e., that occurs at this steplike series of 5f localization transitions. Other noteworthy properties of elemental plutonium include a severely depressed melting temperature with respect to neighboring elements, and striking anomalies especially evident for the $\delta$ cubic close-packed phase when stabilized below room temperature by the addition of gallium or cerium, including a negative thermal expansion and a large temperature-independent magnetic susceptibility.

A full-potential SIC-LDA-LMTO (self-interaction-corrected, local density approximation, linear-muffin-tin-orbital) computation was used to represent the $\delta$ plutonium state. We summarize our results as follows. In understanding the behavior of $\delta$ Pu (fcc with atomic volume expanded $27 \%$ from that in the monoclinic $\alpha$ ground state) it is important to recognize that the lowest energy localized state, as shown by the SIC-LDA total energy 
calculations is that with four rather than five localized $5 f$ electrons, and the next highest state in energy is that with three localized $5 f$ electrons. (Atomic plutonium has six $5 f$ electrons; while in the solid state, plutonium has five $5 \mathrm{f}$ electrons.)

We now consider how the experimental photoemission spectrum of Arko and Joyce at LANL compares to our expectations based on the densities of states for the various SIC-LDA states. In doing this, it is important to bear in mind the $10^{-15}$ second time scale of photoemission. Our expectation is that the observed sharp Kondo resonance peak just below the Fermi level should be best represented by the SIC-LDA density of states for three localized $5 f$ electrons. This is because we expect that state to best capture the turbulent mixing of band and $5 f$ electrons epitomized by the rapid fluctuations between the states with four and three $5 f$ electrons. Indeed, the agreement between the experimental peak and the ab initio calculation is remarkably close. Furthermore, when we examine the corresponding $\left(\mathrm{n}_{f}=3\right) f$-projected density of states (Fig. 5), we see strong evidence for band $5 f$ hybridization, which transfers spectral intensity from nearby energies just below and above the peak into the very strongly hybridized resonant peak seen in the $\mathrm{n}_{f}=3$ total density of states.

In the context of the two (ortho-para)-site model, we expect the remaining nonresonant part of the photoemission spectrum to be reflected in the density of states for the $\mathrm{n}_{f}=4$ SIC-LDA ground state for delta Pu. Indeed, both the double peak falling between about -0.3 ev and -1.1 ev below the Fermi energy and the broad structure between about $-1.1 \mathrm{ev}$ and -2.1 ev are well represented by the SIC-LDA density of states for four localized $5 f$ electrons. Furthermore, comparison with the $f$-projected density of states shows that the double peak between -0.3 and $-1.1 \mathrm{ev}$ is of $5 f$ spectral origin, and the broad shoulder between -1.1 and $-2.1 \mathrm{ev}$ is of band spectral origin. 
Our expectation is that the best overall mean field (long time scale compared to $10^{-12}$ sec) picture of the spectral density from the SIC-LDA calculations is provided by that for five localized $5 f$ electrons, and this indeed is the case. The density for this state is only $2.6 \%$ more than the experimental density further supporting our view that this state provides a very high quality mean field portrayal of delta plutonium after localization has occurred.

\section{Information Access:}

\section{Publications:}

"Development of magnetism in strongly correlated cerium systems: Non-Kondo mechanism for moment collapse”, Physical Review B 62, pp 11533-7. 2000 (E. M. Collins, N. Kioussis, Say Peng Lim, and B.R. Cooper)

"Competition of exchange and crystal field interactions in cerium monopnictides and monochalcogenides”, Journal of Applied Physics 87, pp 5143-5. 2000 (E. M. Collins, N. Kioussis, Say Peng Lim, and B.R. Cooper)

"A Possible Model for $\delta$ Plutonium", B.R. Cooper, Los Alamos Science No. 26, Vol.1, pp 154168. 2000.

"A Theoretical Study of Relativistic Effects in the Electronic Structure of Pu", Physical Review B 61, 12562 (2000). (G.W. Fernando, E.H. Sevilla, and B.R. Cooper).

"Correlation Effects on Stability in Pu Metal and Its Alloys", pp 531-537 in Electron Correlations and Materials Properties (A. Gonis and N. Kioussis, Eds., Plenum Publishing,1999) (B.R. Cooper with P.E.A. Turchi, A. Gonis, N. Kioussis, and D.L. Price).

"From Heavy Fermions to Random-Localized-Site Behavior Via Anderson Localization", Philosophical Magazine B 9ㅗ, 683 (1999). (B.R. Cooper with O. Vogt, Q.G. Sheng, and Y.L. Lin).

"Calculated Lattice Relaxation in Pu-Ga Alloys", Journal of Alloys and Compounds 271-273, 367 (1998). (B.R. Cooper with J.D. Becker, J.M.Wills, and L. Cox).

"Calculated Lattice Relaxation in Pu-Ga", Phys. Rev. B $\underline{58 B}, 5143$ (1998). (B.R. Cooperwith 
J.D. Becker, J.M. Wills, and L. Cox).

\section{Invited Talks at Conferences:}

"The Stabilization of fcc Plutonium: A Solid-State-Solution-Like Phase of Stable and Fluctuating Configuration Plutonium", Plutonium Futores- The Science Conference, Santa Fe, July 10-13, 2000.

"Change with Chemical Environment from Periodic to Spatial-Disordered Magnetic Ordering on a Lattice', Conference on Electronic Structure and Magnetism in Complex Materials, Washington, DC, July 26-28, 2000.

"From Heavy Fermions to Random-Localized-Site $f$-Electronic and Magnetic Behavior Via Anderson Localization", European Conference on Physics of Magnetism 99", Poznan, Poland, June 21-25, 1999.

"The Role of Exchange in Correlated-Electron Dynamics", Czech-US Workshop on Prospects of a Realistic Description of Strong Electron Correlations in Solids, Prague, October 1-6,1999.

"Treating Electronic and Magnetic Properties of Actinide-Based Materials Beyond One-Electron Dynamics", School of Actinide Physics and Chemistry, Uppsala, Sweden, May, 1998.

"Synthesis of Many-Body Theory and Electronic Structure", International Workshop on Electron Correlations and Materials Properties, Heraklion, Crete, Greece, June 28-July 3,1998.

\section{Contributed Presentation at Conferences:}

"Structural Relaxation in Pu-Ga via Full-Potential LMTO Calculations", Actinides 97 International Conference, Baden-Baden, Germany, September, 1997. (B.R. Cooper with J.D. Becker, J.M. Wills, and L. Cox).

"Diffusion of Plutonium into Transition Metallic Alloys and of Transition Metal Species into Plutonium", Eighth Conference on Computational Research on Materials, Lakeview, WV, May, 1998. (B.R. Cooper with S. Beiden).

"Modeling of Diffusion of Plutonium in Other Metals and of Gaseous Species in PlutoniumBased Systems", Environmental Management Workshop, Chicago, July,1998. (B.R. Cooper with S. Beiden).

"Portable Detection and Analysis of Plutonium Content", Workshop on Environmental Management Science: Integration with End User Needs, Savannah River Site, November, 1998. (B.R. Cooper with D. Lederman).

"Modeling of Diffusion of Plutonium", Workshop on Environmental Management Science: Integration with End User Needs, Savannah River Site, November, 1998. (B.R. Cooper with 


\section{S.Beiden).}

"Modeling of Interdiffusion of Plutonium and Other Metals", Materials Research Society Meeting, Boston, November,1998. (B.R. Cooper with S. Beiden).

"Anomalous Electronic Behavior and Relationship to Thermostructural Behavior of Light Actinides", American Physical Society Meeting, Atlanta, March, 1999.

"Electronic Structure of Alpha and Delta Plutonium", American Physical Society Meeting, Atlanta, March,1999. (B.R. Cooper with N. Kioussis, P.E.A. Turchi, A. Gonis and D.L.Price).

"Equilibrium Lattice Volume of fcc Pu", American Physical Society Meeting, Atlanta, March, 1999. (B.R. Cooperwith E.H. Sevilla and G.W. Fernando).

"Random $5 f$ Localization and the fcc Transition and Depression of Melting Temperature in Plutonium, 29th Journees des Actinides Conference, Luso, Portugal, April 1999.

"Theory and Simulation of Phase Transitions in Plutonium", American Physical Society Meeting, Minneapolis, March, 2000. (with A. Setty and D.L. Price).

"Between Periodic $5 f$ Localization Spatially-Disordered $5 f$ Localization, and $5 f$ Delocalization in Uranium and Plutonium Systems", 30th Jourees des Actinides, Dresden, Germany, May 4-6, 2000. (with Y.L. Lin and A. Setty).

"Ab Initio Predicting of Phase Transitions in Plutonium", Tenth Conference on Computational Research on Materials, Morgantown, WV, May 17-19, 2000 (with A. Setty and D.L. Price).

"Theory and Simulation of Phase Transitions in Plutonium", Conference on Electronic Structure and Magnetism in Complex Systems, Washington, DC, July, 2000 (with A. Setty and D.L. Price).

"Stability of Expanded-Volume fcc Phases of Cerium and Plutonium as Homogeneously Disordered Solids", Meeting of the American Physical Society, Seattle, March, 2001 (with D. Djajaputra).

"Self-Interaction_Corrected Calculation of the Energetics of Stabilization and Density of the Expanded-Volume (Delta) fcc Phase of Plutonium", Journe'es des Actinides Conference, St. Malo, France, April, 2001 (with A. Setty).

"Stability of Expanded Volume fcc Plutonium", Eleventh Conference on Computational Research on Materials, Lakeview, WV, May, 2001 (with A. Setty). 